Research paper

\title{
Genotoxicity, biodistribution and toxic effects of silver nanoparticles after in vivo acute oral administration
}

\author{
Laura Narciso $^{\mathrm{a}, \mathrm{d}}$, Lucia Coppola ${ }^{\mathrm{a}, \mathrm{b}}$, Gabriele Lori ${ }^{\mathrm{a}, \mathrm{c}}$, Cristina Andreoli ${ }^{\mathrm{d}}$, Andrea Zjino ${ }^{\mathrm{d}}$, \\ Beatrice Bocca $^{\mathrm{d}}$, Francesco Petrucci ${ }^{\mathrm{d}}$, Antonio Di Virgilio ${ }^{\mathrm{e}}$, Andrea Martinelli ${ }^{\mathrm{e}}$, Antonella Tinari ${ }^{\mathrm{a}}$, \\ Francesca Maranghi ${ }^{\mathrm{a}, *}$, Roberta Tassinari ${ }^{\mathrm{a}}$ \\ ${ }^{a}$ Center for Gender-Specific Medicine, Istituto Superiore di Sanità, Rome, Italy \\ ${ }^{\mathrm{b}}$ Department of Physiology and Pharmacology V. Erspamer, Sapienza University of Rome, Rome, Italy \\ ${ }^{\mathrm{c}}$ Department of Science, University of Rome "ROMA TRE", Rome, Italy \\ ${ }^{\mathrm{d}}$ Environment and Health Department, Istituto Superiore di Sanità, Rome, Italy \\ ${ }^{\mathrm{e}}$ Experimental Animal Welfare Sector, Istituto Superiore di Sanità, Rome, Italy
}

\section{A R T I C L E I N F O}

Editor: Philip Demokritou

Keywords:

Food additive

OECD guideline 489

CD1 mice

Comet assay

Micronucleus assay

\begin{abstract}
A B S T R A C T
In the last years, silver nanoparticle (AgNP) use is increased due to the presence in several consumer products, including cosmetics and food packaging, for their antimicrobial activities. Silver in its elemental form is authorised as food additive E174 and EFSA noted that approximately a 20\% of AgNPs are released from confectionary pearls. Toxicological assessment of E174 performed by EFSA concluded that the available information was insufficient to assess the safety for consumers and one of the major issue was the in vivo genotoxic potential. Aim of the present study was to provide data on in vivo genotoxicity of AgNPs by Alkaline Comet Assay according to the OECD Test Guideline (TG) 489 - and by Micronucleus Assay. AgNP dispersions (20 nm) were orally administered to male and female mice for three days at 50,150, $300 \mathrm{mg} / \mathrm{kg}$ bw per day on the basis of the OECD TG 489. AgNP dispersions were fully characterized. Comet assay was performed on blood, liver, spleen, duodenum and kidney, and Micronucleus assay on spleen lymphocytes, to evaluate the genotoxic potential. Biodistribution and histopathological assessment were performed. AgNPs accumulated in duodenum as first contact site and transferred to other target tissues; in liver and duodenum they were free in the cytoplasm or included in organelles but never in the nucleus, as detected by Transmission Electron Microscope analysis. No genotoxic or tissue damage was recorded by both assays in all the tested tissues. The in vivo genotoxicity data supported a more comprehensive risk assessment of AgNPs.
\end{abstract}

\section{Introduction}

Consumption of colloidal silver nanoparticles (AgNPs) as dietary supplement for the treatment of specific diseases and various infections has been known for decades (Kim et al., 2007). In the last years, AgNP use is further increased due to the inclusion in numerous consumer products, including cosmetics, food packaging, apparel, footwear, paints, wound dressings, appliances, mainly for their antimicrobial activities (Calderon-Jimenez et al., 2017; Ullah et al., 2018).

Moreover, silver in its elemental form is authorised as food additive E174 in the European Union (EU) and used as food colour (EFSA Panel on Food Additives and Nutrient Sources added, to Food, 2016). Approximately a $20 \%$ of E174 can be released from confectionary pearls as AgNPs - median diameter of $23.3 \mathrm{~nm}$ (Verleysen et al., 2015), whereas other studies showed that the fraction of AgNPs (size range of $60-300 \mathrm{~nm}$ ) released from plastic food packaging items is very low and depends on the food composition (Choi et al., 2018). Indeed, ingestion is one of the most relevant route of human exposure to AgNPs, with inhalation and dermal absorption giving also a significant contribution (EFSA Panel on Food Additives and Nutrient Sources added, to Food, 2016; Blanco et al., 2018). AgNPs are unstable and release ions through reaction with oxygen and protons or with pre-existing oxide films in fluid media, and the oxidative dissolution is influenced by $\mathrm{pH}$, temperature, coatings and ligands (Liu and Hurt, 2010; Liu et al., 2012; Behra et al., 2013); ion formation strongly contributes to biological activity of AgNPs keeping them potentially harmful to humans (Hartemann et al., 2015).

Toxicological assessment of E174, which included the AgNP

\footnotetext{
* Corresponding author at: Istituto Superiore di Sanità, Viale Regina Elena, 299, 00161 Rome, Italy.

E-mail address: francesca.maranghi@iss.it (F. Maranghi).
} 


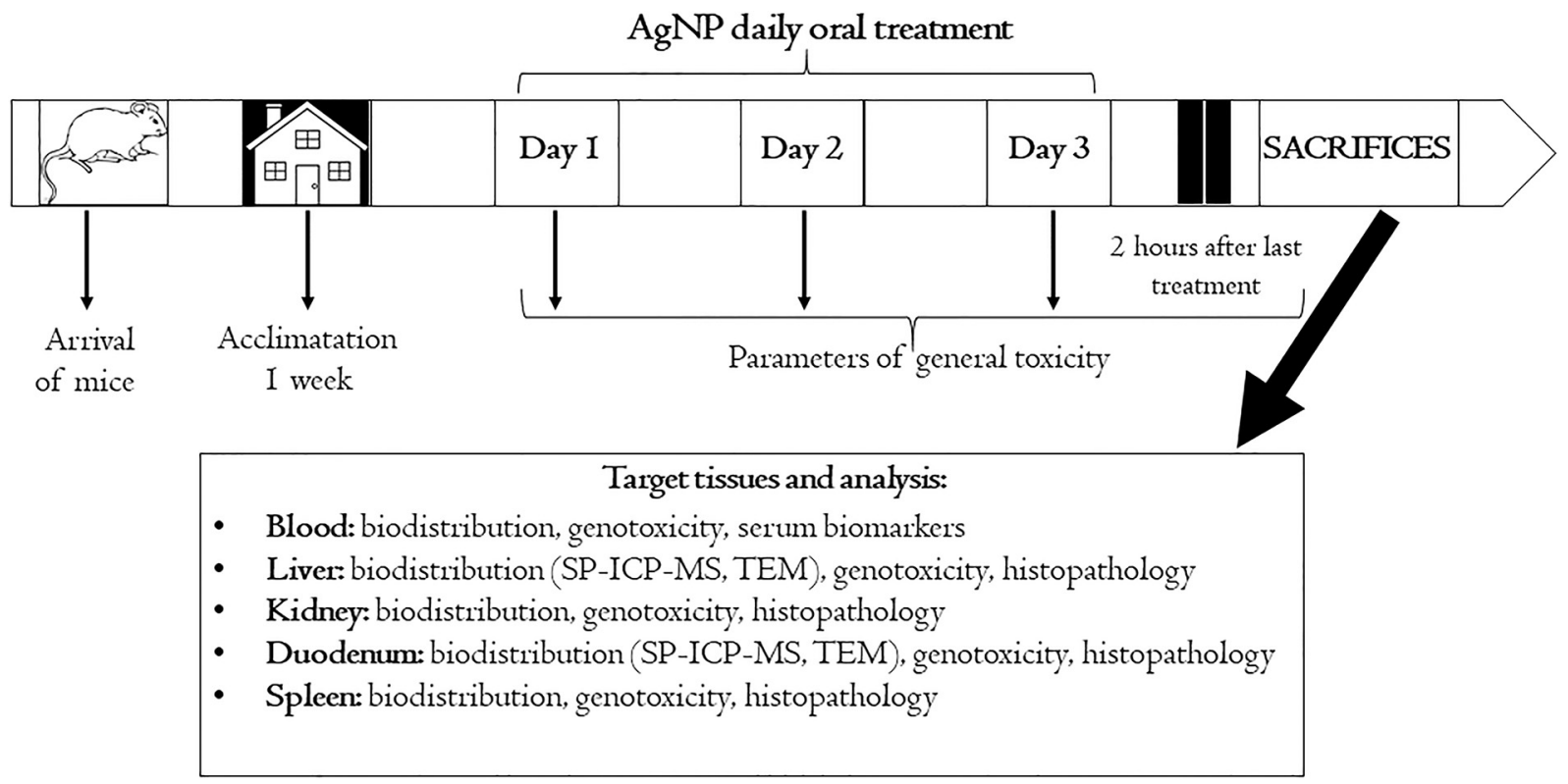

Fig. 1. Experimental design of male and female CD1 mice orally exposed by gavage for 3 days with 50,150 and 300 mg/kg bw per day of AgNPs.

evaluation, was performed by European Food Safety Authority (EFSA) in 2016; EFSA concluded that the available information was insufficient to assess the safety of $\mathrm{Ag}$ as food additive and the major problems are linked to chemical identification and characterization in terms of quantity of NPs and ion release, and data on the material used in the available toxicological studies (EFSA Panel on Food Additives and Nutrient Sources added, to Food, 2016; EFSA Panel on Contaminants in the, Food Chain, 2015). Similar opinion was expressed by the Scientific Committee on Consumer Safety (SCCS) concerning the use of AgNPs in cosmetics and consumer products (SCCS. Scientific Committee on Consumer Safety, 2018).

One of the major issue in hazard identification of AgNPs is the genotoxic potential - identified by EFSA as key step in the safety evaluation of NPs present in food and feed (EFSA Scientific Committee et al., 2018). AgNPs showed to be genotoxic in various in vitro test systems, but the in vivo genotoxicity studies, including by the oral exposure, provided less conclusive data; indeed, a definitive evaluation of the possible genotoxic hazard associated with oral exposure to AgNPs has not yet achieved.

Aim of the present study was to provide data on in vivo genotoxicity of AgNPs by Alkaline Comet Assay according to the OECD Test Guideline 489 (OECD, 2016a). The Comet assay is frequently used in nano-genotoxicology (Glei et al., 2016; Karlsson et al., 2015) to identify substances that cause DNA damage, and alkaline conditions ( $>\mathrm{pH} 13$ ) detect single and double stranded breaks. The strand breaks may have different fate (repaired, be lethal to the cell or be fixed in a mutation) and could cause chromosomal damages associated with human diseases (OECD, 2016a; Narciso et al., 2016; Carlessi et al., 2014; Caldecott, 2008).

Moreover, the potential induction of chromosome damage by in vivo exposure to AgNPs was assessed by the Micronucleus test (Hayashi, 2016) in lymphocytes derived from spleen (OECD, 2016b), as representative of potential effect at whole organism level.

AgNPs of $20 \mathrm{~nm}$ of diameter - which represents the relevant dimension for human exposure through food (Verleysen et al., 2015) were orally administered for 3 days to male and female adult CD-1 mice. The dose levels were selected according to literature data (Boudreau et al., 2016) and with the aim of identifying genotoxic effects (Patlolla et al., 2015a; Martins Jr et al., 2017; Asare et al., 2016). The characterization of the dispersions used for animal treatment, the biodistribution of AgNPs in selected target tissues by different techniques as Single Particle-inductively coupled plasma mass spectrometry (SP-ICP-MS) and Transmission Electron Microscope (TEM), general toxicity, liver and kidney serum biomarker analysis and histopathological examinations of target tissues were performed in order to provide data for a more comprehensive AgNP hazard identification.

\section{Material and methods}

\subsection{Characterization of AgNP dispersion}

AgNPs dispersed in distilled water were purchased from HiQ-Nano (Arnesano, Lecce, Italy) and the characterization of the dispersion was provided by the manufacturer. Briefly, TEM images of AgNPs were recorded by a JEOL Jem 1011 microscope. TEM samples were prepared by dropping a dilute solution of NPs in water on carbon-coated copper grids (Formvar/Carbon 300 Mesh Cu). Dynamic Light Scattering (DLS) measurements were performed on a Zetasizer Nano ZS90 (Malvern, USA). The optical absorbance spectra of AgNPs were measured with a Spectrophotometer Nanodrop 2000c at a resolution of $1 \mathrm{~nm}$. Measurements were made at $25{ }^{\circ} \mathrm{C}$ in aqueous solutions at $\mathrm{pH} 7$ and in these conditions the formation of silver ions $\left(\mathrm{Ag}^{+}\right)$was minimal (De Matteis et al., 2015).

\subsection{Animal experimental design}

The study was conducted according to the Directive 2010/63/EU, the Italian Legislative Decree n. 26 of 4 March 2014, the OECD Principles of Good Laboratory Practice (GLP) and on the basis of the OECD Test Guideline 489 (Glei et al., 2016). Moreover, the quality criteria for in vivo toxicity studies performed with nanomaterials, according to Fernández-Cruz et al. (2018), were met. Fig. 1 shows the scheme of experimental design. Fourty six adult male and female outbred CD1 mice $(31.4 \pm 3.4 \mathrm{~g}$ male mice and $27.4 \pm 1.7 \mathrm{~g}$ female mice, $5 / 6$ weeks old) were purchased from Charles River (Calco, Lecco, Italy). They were housed two/cage, under standard laboratory conditions $\left(22 \pm 0.5{ }^{\circ} \mathrm{C}, 50-60 \%\right.$ relative humidity, $12 \mathrm{~h}$ of dark-light alternation with 12-14 air changes per hour) with water and food (2018 Global Diet purchased from Mucedola, Milan, Italy) available ad libitum. After 1 week of acclimatization, mice were divided into four treatment groups (5 mice/sex/group): 
1) Control, vehicle only [distilled water] $=$ CTRL

2) $50 \mathrm{mg} / \mathrm{kg}$ body weight (bw) per day = Ag50

3) $150 \mathrm{mg} / \mathrm{kg}$ bw per day $=\mathrm{Ag} 150$

4) $300 \mathrm{mg} / \mathrm{kg} / \mathrm{bw}$ per day Ag300.

An additional group of 3 mice/sex was treated with $100 \mathrm{mg} / \mathrm{kg}$ bw per day of methyl methanesulphonate (MMS) as positive control for genotoxicity assays. All mice were treated once a day per os by gavage for 3 consecutive days. In order to ensure the stability of test compound during the treatment, dispersions of AgNPs were maintained at $4{ }^{\circ} \mathrm{C}$ throughout the duration of the experiment and mixed immediately before use according to the manufacturer's instructions. The concentration of dispersions was calculated in order to supply volumes of $2 \mathrm{ml} / 100 \mathrm{~g}$ of bw. Mice were checked daily for their health status, individual bw and food consumption were recorded; dose levels of AgNPs were adjusted according to the weight gain for each animal during the treatment. Two hours after the last treatment (day 3), mice were anaesthetised with a gaseous solution of isoflurane and blood samples were collected by intracardiac puncture. $100 \mu \mathrm{l}$ of blood were collected into EDTA-coated microtainers (Becton Dickinson, CA, USA) and processed for biodistribution evaluation and Comet assay. The remaining blood was used for the determination of serum hepatic and renal biomarkers. Subsequently, mice were sacrificed by $\mathrm{CO}_{2}$ inhalation and liver, kidney, duodenum and spleen were excised and weighed. Duodenum was not weighed following excision due to the difficulty in the standardization of the tissue portion trimmed at sacrifice.

Biodistribution analysis and Comet assay were performed in blood, liver, kidney, duodenum and spleen. Micronucleus assay was performed on lymphocytes derived from the spleen. Histopathological analyses were performed on liver, kidney, duodenum and spleen. TEM analysis was performed on liver and duodenum.

\subsection{Tissue homogenate preparation}

A portion of liver, spleen, duodenum and one kidney were put in a Petri dish, washed with cold mincing buffer (Phosphate buffered saline, PBS, containing $25 \mathrm{mM}$ Na2EDTA and 10\% Dimethyl Sulfoxide, DMSO) and kept on ice. Cells of liver and kidney were mechanically isolated (Medimachine ${ }^{\circledR}$, Becton Dickinson). Duodenum was rinsed with mincing buffer, cut longitudinally and scraped with a spatula. Spleen was minced in $3 \mathrm{ml}$ di RPMI medium 1640 (GIBCO). Cellular suspension (except spleen) was divided in 2 aliquots: one for biodistribution analysis and the second, used for Comet and Micronucleus assays, was flowed through $50 \mu \mathrm{m}$ filter (Filcons, BD Bioscences) and centrifuged $8 \mathrm{~min}$ at 500 round per minute (rpm) at $4{ }^{\circ} \mathrm{C}$; pellets were resuspended in cold PBS. Cells derived from spleen were suspended in $3 \mathrm{ml}$ of RPMI 1640, stratified on $2 \mathrm{ml}$ di Hystopaque1077 and centrifuged for $30 \mathrm{~min}$ at $1400 \mathrm{rpm}$ at $4{ }^{\circ} \mathrm{C}$. Buffy coat was collected and suspended in PBS $1 \times$ and centrifuged at $1000 \mathrm{rpm}$ for $10 \mathrm{~min}$ at $4{ }^{\circ} \mathrm{C}$; cell pellet was counted and divided in two aliquots: $1 \times 10^{6}$ cells were used for Comet assay and remaining cells were cultured in RPMI 1640 plus $15 \%$ of inactivated Foetal Calf Serum (Hyclone) for Micronucleus assay. Two or more cultures depending on the number of splenocytes were set up with at least $2 \times 10^{6}$ cells for each animal.

\subsection{SP-ICP-MS analysis}

AgNPs were analyzed by SP-ICP-MS using an iCAP-Q ICP-MS (Thermo Fisher, Bremen, Germany), with Meinhard nebulizer and cyclonic chamber. For the Ag quantification the isotope $m / z 107$ was used with a dwell time of $5 \mathrm{msec}$ and an analysis time of $60 \mathrm{~s}$ per sample. The operating conditions were reported in previous papers (Bocca et al., 2017; Bocca et al., 2018).

Tissue homogenates and blood samples were subjected to alkaline extraction using $1.5 \mathrm{ml}$ of tetramethylammonium hydroxide (TMAH) at $25 \% \mathrm{w} / \mathrm{w}$, sonicated for $1 \mathrm{~h}$ in ultrasonic bath and then were left at room temperature for $24 \mathrm{~h}$. Subsequently, the solutions were filled up to $15 \mathrm{ml}$ with a $0.1 \%$ aqueous solution of Triton X-100. Before the analysis by SP-ICP-MS the extraction solutions were further properly diluted with ultrapure deionized water.

Recovery and repeatability were carried out in order to verify the validity of the SP-ICP-MS method by comparing the concentration (as number of particles/ml) of the reference standard of AgNP at $20 \mathrm{~nm}$ (Hiq-nano) in water and in organ and blood extracts solutions. To this end, the reference standard of AgNP was added to tissue and blood samples of untreated mice (CTRL) before the TMAH extraction; the samples were then subjected to the extraction procedure described above and analyzed by SP-ICP-MS. The tests were performed on 3 independent samples. The stability of AgNPs in samples was evaluated by comparing the variations in size (in $\mathrm{nm}$ ) of the reference standard in water and on extracted samples.

\subsection{TEM analysis}

Small portion of liver and duodenum of one Ag300 and CTRL mouse/sex was fixed in $2.5 \%$ glutaraldehyde in cacodylate buffer $0.1 \mathrm{M}, \mathrm{pH} 7.2$, washed and fixed in $1 \% \mathrm{OsO}_{4}$. After washing, fixed specimens were dehydrated through a graded series of ethanol solutions and embedded in Agar 100 resin (Agar Scientific, Essex, UK). Ultrathin sections were collected on 200-mesh copper grids and counterstained with uranyl acetate and lead citrate. Sections were observed with a Philips $208 \mathrm{~s}$ electron microscope at $100 \mathrm{kV}$.

\subsection{Liver and kidney serum biomarkers}

The following functional biomarkers were measured in serum of AgNPs treated and CTRL mice of both sexes: Glutamic Oxaloacetic Transaminase (GOT), Glutamic Pyruvic Transaminase (GPT), Blood Urea Nitrogen (BUN) and plasma Creatinine (CREA).

Blood samples collected prior to the sacrifice were left to coagulate at room temperature for $1 \mathrm{~h}$ and then centrifuged for $15 \mathrm{~min}$ at $1000 \mathrm{~g}$ twice in a cooled bench-top centrifuge (Microlite Microfuge, Thermo Electron Corporation). After centrifugation, the serum was stored at $-80{ }^{\circ} \mathrm{C}$ until use. GOT, GPT, CREA and BUN were measured by the automatic analyser Keylab liquid chemical analyser (BPC Biosedsrl, Italy) (Tassinari et al., 2019).

\subsection{Genotoxicity endpoints}

\subsubsection{Comet assay}

The protocol used in the present study was obtained by a modified protocol of alkaline Comet assay from Singh (Singh et al., 1988). After mixing $30 \mu \mathrm{l}$ of blood or 20,000 cells $/ \mu 1$ with $195 \mu \mathrm{l}$ of $0.7 \%$ low melting point agarose (Bio-Rad Lab., Hercules, CA, USA), $75 \mu \mathrm{l}$ were seeded on slides pre-coated with $1 \%$ normal melting point agarose (BioRad). Slides were covered with glass coverslips and kept on a cold plate until gel solidification.

Two slides per tissue were prepared for each animal; the slides without coverglass were put into lysis solution $(2.5 \mathrm{M} \mathrm{NaCl}, 0.1 \mathrm{M}$ Na2EDTA, $10 \mathrm{mM}$ Tris pH 10, extemporarily added with $10 \%$ DMSO and $1 \%$ Triton X-100) and kept overnight at $4{ }^{\circ} \mathrm{C}$. The slides were placed in an electrophoresis tank and DNA was unwinded at $4{ }^{\circ} \mathrm{C}$ for $20 \mathrm{~min}$ in fresh electrophoresis buffer (0.3 M NaOH, $1 \mathrm{mM} \mathrm{Na2EDTA,} \mathrm{pH}>13$ ). Electrophoresis was carried out in the dark at $4{ }^{\circ} \mathrm{C}$ for $20 \mathrm{~min}, 21 \mathrm{~V}$ $(1.4 \mathrm{~V} / \mathrm{cm}$ corresponding to voltage/distance of platform on which the slides are placed (Azqueta et al., 2011), $300 \mathrm{~mA}$ ), then the slides were washed 3 times for $5 \mathrm{~min}$ into a neutralizing solution $(0.4 \mathrm{M}$ Tris- $\mathrm{HCl}$, $\mathrm{pH}$ 7.5), fixed with cold ethanol for $5 \mathrm{~min}$ and dried for conservation. Coded slides were stained with Gel RED Nucleic Acid Gel Stain according to supplier instructions (Biotium, Fremont, CA) and examined with a Nikon fluorescence microscope (ECLIPSE 80i) by a computerized image analysis system (LUCIA Comet Assay $^{\mathrm{TM}}$, Laboratory Imaging, 
Table 1

AgNPs characterization in water.

\begin{tabular}{lll}
\hline AgNP diameter by TEM (nm) & $\begin{array}{l}\text { AgNP diameter by DLS } \\
(\mathrm{nm})\end{array}$ & $\begin{array}{l}\text { AgNP by UV-Vis } \\
(\mathrm{nm})\end{array}$ \\
\hline $20.7 \pm 3.0$ & $23.5 \pm 0.7$ & 393 \\
\hline
\end{tabular}

Prague). For all cells derived from different tissues a total of 150 cells from two different slides were scored for each tissue/animal. For cells derived from spleen: one hundred and fifty comets were randomly scored using a dedicated image analysis system (IAS2000; Delta Sistemi, Rome, Italy) connected to a fluorescence microscope (Leica, Wetzlar, Germany) equipped with appropriate filters (N2.1) and connected to a computer through a charge-coupled device camera. According to OECD guideline 489 (Organisation for Economic Co-operation and Development. 2014), the parameters considered were: the percentage of total DNA in the comet tail (tail intensity, \%T.I.) and the hedgehogs or ghosts (\%G.), identified by visual inspection or when their tail intensity value was higher than $80 \%$. Media of medians of each group were considered to analyze DNA damage and error standard of the mean (Maranghi et al., 2018).

\subsubsection{Micronucleus assay}

Isolated splenocytes were cultured in cell culture tubes containing RPMI 1640 medium (Gibco), 15\% of inactivated Foetal Calf Serum (Gibco), 1\% penicillin/streptomycin (5000 IU and $5000 \mathrm{mg} / \mathrm{ml}$, respectively) (GIBCO) and $5 \mu \mathrm{g} / \mathrm{ml}$ Concanavalin A (Sigma). Cultures were incubated at $37{ }^{\circ} \mathrm{C}$ in a $5 \% \mathrm{CO}_{2}$ atmosphere. After $24 \mathrm{~h}$ of incubation, cytochalasin B (Sigma) was added at the final concentration of $5 \mu \mathrm{g} / \mathrm{ml}$. After additional $20 \mathrm{~h}$ of incubation, cells were harvested on slides by cytocentrifuge (Shandon II). After air-drying, slides were fixed in methanol and stained in $10 \%$ Giemsa. For each sample derived from negative and positive control groups, and groups exposed to the 3 dose levels of AgNPs, the frequency of mononucleated, binucleated and multinucleated cells on 1000 cells was recorded to calculate the Replication Index (RI) according to the following formula:

$\mathrm{RI}=\left(\mathrm{n}^{\circ}\right.$ binucleated cells $)+\left(2 \times \mathrm{n}^{\circ}\right.$ multinucleated cells $) / \mathrm{n}^{\circ}$ total cells

The frequency of binucleated cells bearing micronucleus was calculated on 1000 total binucleated cells. Results are expressed as mean values of 5 animals for each group.

\subsection{Histopathological and histomorphometrical analysis}

Immediately after the sacrifice, to avoid any post-mortem artefact, a portion of liver, spleen, duodenum and one kidney were fixed in $10 \%$ buffered formalin and stored in $80 \%$ ethyl alcohol. Specific care was paid to easily perishable tissues, e.g. spleen. Tissues were embedded in paraffin, cut into $5-\mu \mathrm{m}$ sections and stained with haematoxylin and eosin before examination under a light microscope (Nikon Microphot FX) with different lenses.

Quantitative histomorphometrical analyses were performed on spleen, duodenum and kidney of CTRL and treated mice. Briefly, slides of selected tissues were examined by means of an image analysis system (Nis-Elements D) applied to an optical microscope (Nikon Microphot FX).

Duodenum: using a $10 \times$ lens, the height of the mucosal tunic, the width and height of intestinal villi were measured (25 villi/slide were random chosen) (Tassinari et al., 2015).

Spleen: using a $2 \times$ lens, ratio between white and red pulp areas was measured on whole transversal section (Tassinari et al., 2014).

Kidney: using a $10 \times$ lens, diameter and area were measured on 100 glomeruli (Yabuki et al., 1999). The glomerular volume was calculated according to procedure described by Romero (Romero et al., 1999).

\subsection{Statistical analysis}

Statistical analysis was performed with JMP 10 (SAS Institute Inc., Cary, NC, USA). The GraphPad Prism 6.0 software was used to perform all graphics. Non parametric Kruskal-Wallis test was applied to all the continuous variables to test the statistical significance of the differences among groups, followed by the post-hoc Dunn's test comparison, where appropriate. For genotoxicity data, One-way ANOVA was performed followed by the post-hoc Dunnett's test comparison among treated groups and controls, where appropriate. For categorical variables (histological endpoints), data were expressed as proportions of quantal data; pairwise comparisons of treated groups with CTRL group were analyzed by means of the two-tailed Fisher exact test. To identify dose-response trends, the Mantel-Haenszel $\chi 2$ trend test was used. Significance level was set at $\mathrm{P}<0.05$ for all the analyses.

\section{Results}

\subsection{Characterization of AgNP dispersions}

AgNP dispersion was physically and chemically characterized by the manufacturer in water using TEM, DLS, Z-Potential, UV-vis. The main characteristics are summarized in Table 1. Briefly, TEM analysis showed that AgNPs have a good spherical morphology with a size of approximately $20 \mathrm{~nm}$ of diameter. Monodispersion was confirmed in solution by DLS, showing a peak corresponding to $20 \mathrm{~nm}$; the Z-potential of AgNPs was $-16 \mathrm{mV}$, a sharp plasmon peak at c.a. $400 \mathrm{~nm}$ indicated highly monodispersed NPs (De Matteis et al., 2015).

\subsection{General toxicity}

No adverse signs of toxicity or mortality were observed in Ag50, Ag150 and Ag300 treated mice. Body weight gain, feed consumption, absolute and relative organ weight were unaffected by the treatment both in female and male mice (Supplementary data, Table 1S).

\subsection{AgNP biodistribution in organs and blood}

The SP-ICP-MS method used to analyze dimensional distributions (nm) and concentration (particles/ml) of AgNPs in organs and blood was firstly validated by the measurements of stability, recovery and repeatability, in order to guarantee that no aggregation/agglomeration or dissolution of particles was present in the different matrices after TMAH extraction (Table 2).

In Fig. 2, the number of AgNPs/ml tissue in blood (A), liver (B), kidney (C), duodenum (D) and spleen (E) of male and female mice is shown. Interestingly, differently from female mice, in spleen of male mice no statistically significant differences in treated groups were recorded compared to CTRL. Moreover, dose-response trend and comparable curves between sexes were present in AgNP biodistribution in blood, liver, kidney and spleen with higher AgNP concentration in female mice in blood and kidney, and in male mice in liver and spleen. In duodenum, no dose-response trend was present in both sexes.

Table 2

Validation data of the SP-ICP-MS analysis of AgNPs in water, organs and blood (values are an average of 3 independent samples).

\begin{tabular}{llll}
\hline & Water & Organs & Blood \\
\hline Stability (diameter, nm) & $22.7 \pm 2.93$ & $23.6 \pm 3.3$ & $23.8 \pm 3.6$ \\
Recovery (\%) & 101 & 91.8 & 106.6 \\
Repeatability (\%) & 2.6 & 3.7 & 6.0
\end{tabular}



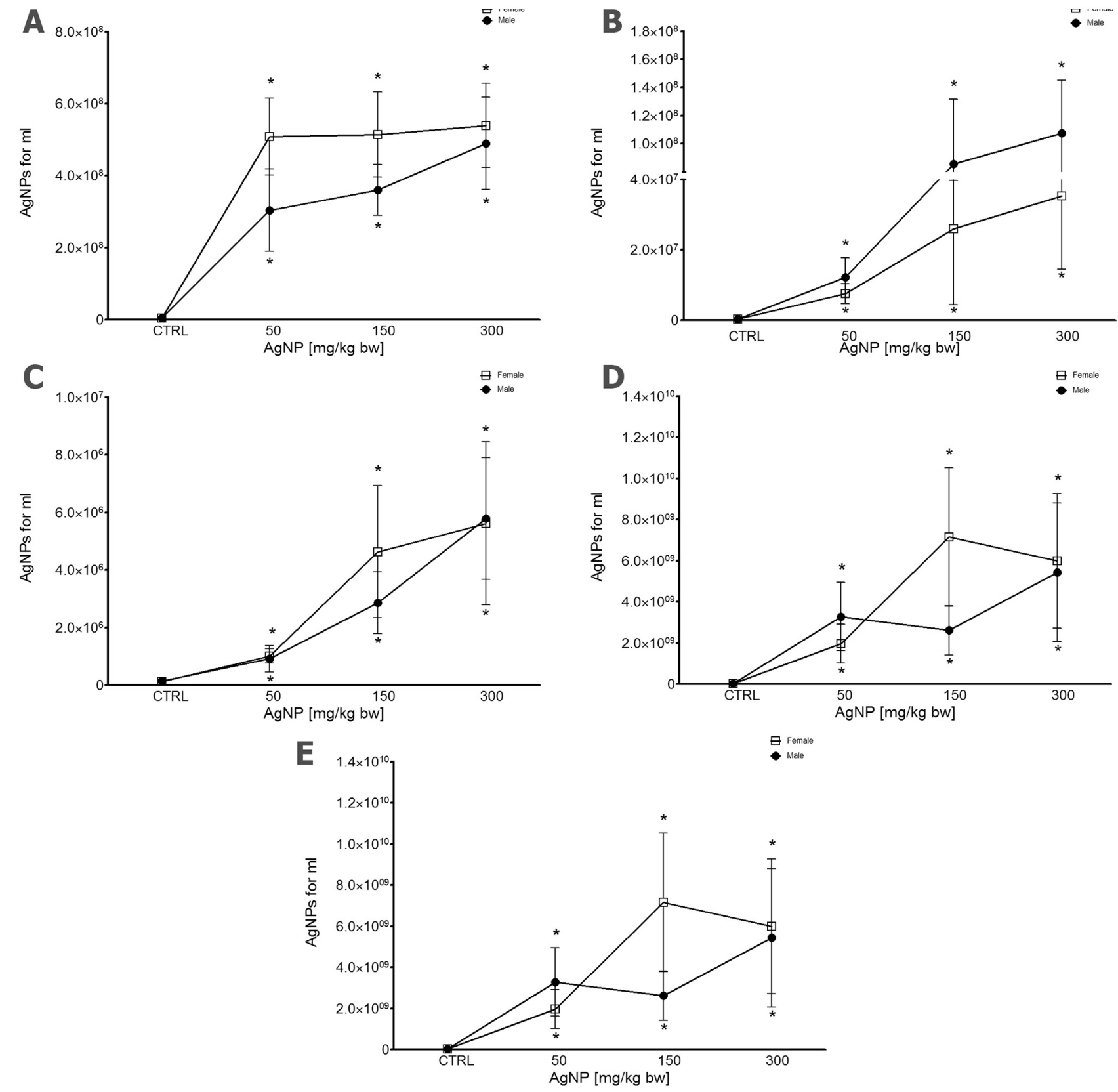

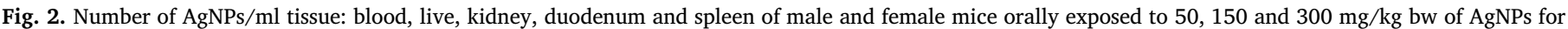
3 days and measured by SP-ICP-MS. * $\mathrm{P}<0.05$.

\subsection{TEM analysis}

TEM images of duodenum and liver are shown in Figs. 3 and 4. Hepatic and duodenal cells of Ag300 treated male and female mouse showed no pathological ultrastructural changes in nuclei, cytosol and organelles, and cells from CTRL tissues were comparable with treated tissues. In liver, AgNPs were located either inside membrane bound structures or dispersed in the cytosol (Fig. 3). In the intestinal cells, AgNPs were located inside membrane bound structures or dispersed in cytosol along microvilli and the presence was comparable in treated and CTRL mice (Fig. 4). Both in liver and in duodenum, no AgNPs were detected in the nucleus (Figs. 3 and 4). Interestingly, both in liver and duodenum of female mice, AgNPs appeared to be more abundant and better distributed with well-defined membrane in comparison to the same tissues of male mice.

\subsection{Liver and kidney serum biomarkers}

Liver serum biomarkers showed, in female mice, significantly reduced levels of GOT and GPT in Ag50 and in Ag150, respectively. No statistical significant alterations were present in male mice (Fig. 5).

Kidney serum biomarkers showed, in male mice, significantly increased levels of BUN in Ag300-No effects were recorded in female mice (Fig. 6).

\subsection{Genotoxicity endpoints}

\subsubsection{Comet assay}

No statistically significant increased DNA damage was observed in blood, liver, kidney, spleen and duodenum of male and female mice exposed to AgNPs in comparison to CTRL. As expected, in mice treated with MMS (positive control) the $\%$ mean of T.I. and \% G. were 


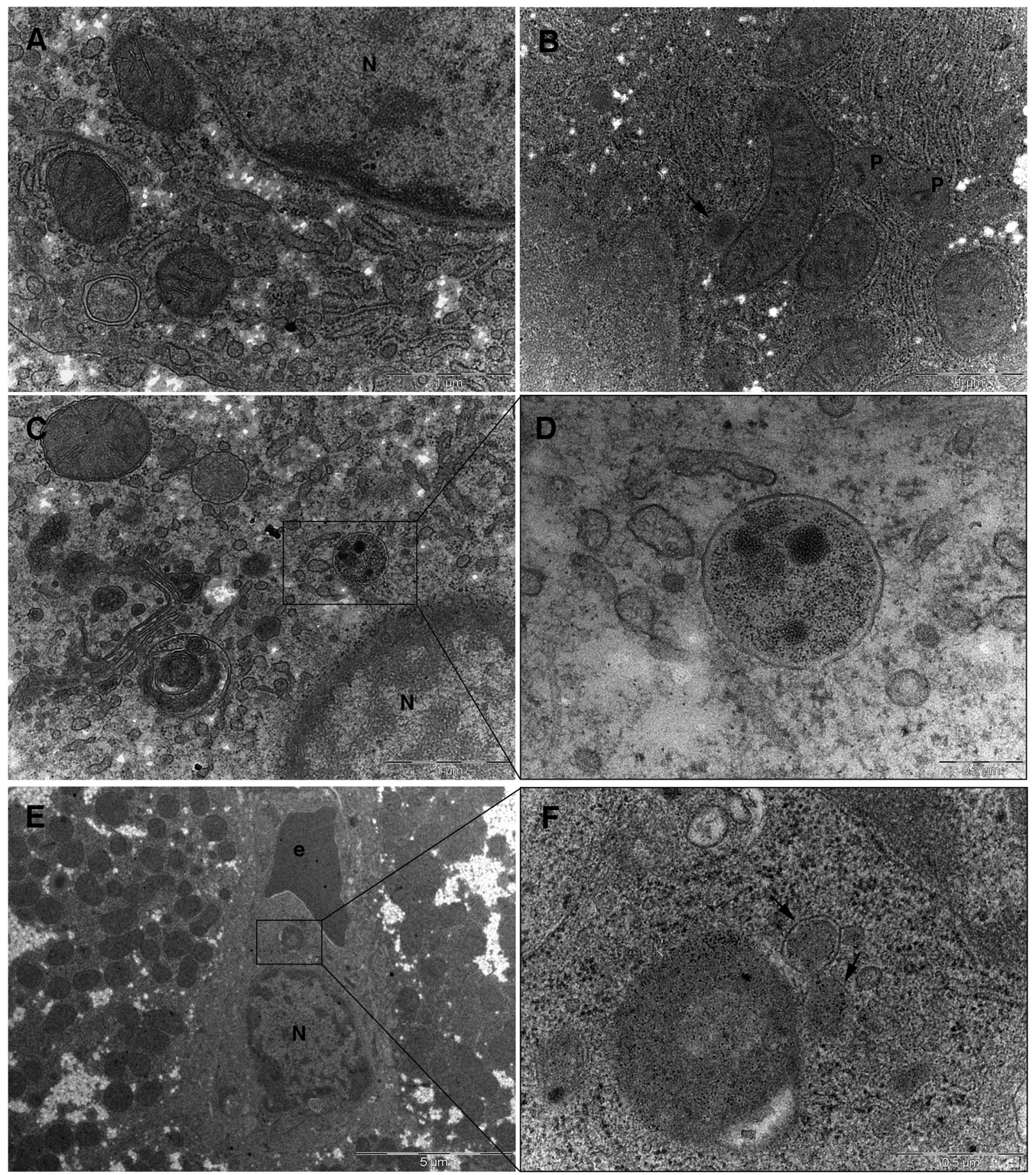

Fig. 3. TEM images of liver of female and male mice orally exposed to $300 \mathrm{mg} / \mathrm{kg}$ per day of AgNPs for 3 days. A) Liver of control mouse (N, nucleus). B) Liver of male mice showing an accumulation of nanoparticles next to mitochondria (arrow) (P, peroxisomes). C) Micrograph of liver of female mice displaying a large amount of nanoparticles within well-defined membrane bound structure (D, enlargement of squared area in C). E) Liver of male mice in which nanoparticles can be observed within two membrane-bound structures (F, arrows) as well as in an electron dense area (F, enlargement of squared area in E).

significantly higher than in CTRL (Table 3).

\subsubsection{Micronucleus assay}

The oral exposure to AgNPs didn't increase the percentage of micronuclei in lymphocytes of spleen in male and female mice, although a slight, dose-related increased frequency was present in Ag150 and Ag300 female mice (Fig. 7).

\subsection{Histopathological and histomorphometrical analysis}

No significant differences were present in liver, kidney, duodenum and spleen of AgNPs treated male and female mice compared to CTRL (Supplementary data, Table 2S).

\section{Discussion}

The present study showed that AgNPs $20 \mathrm{~nm}$ of diameter, relevant dimension for human oral exposure, were present in blood, duodenum, 

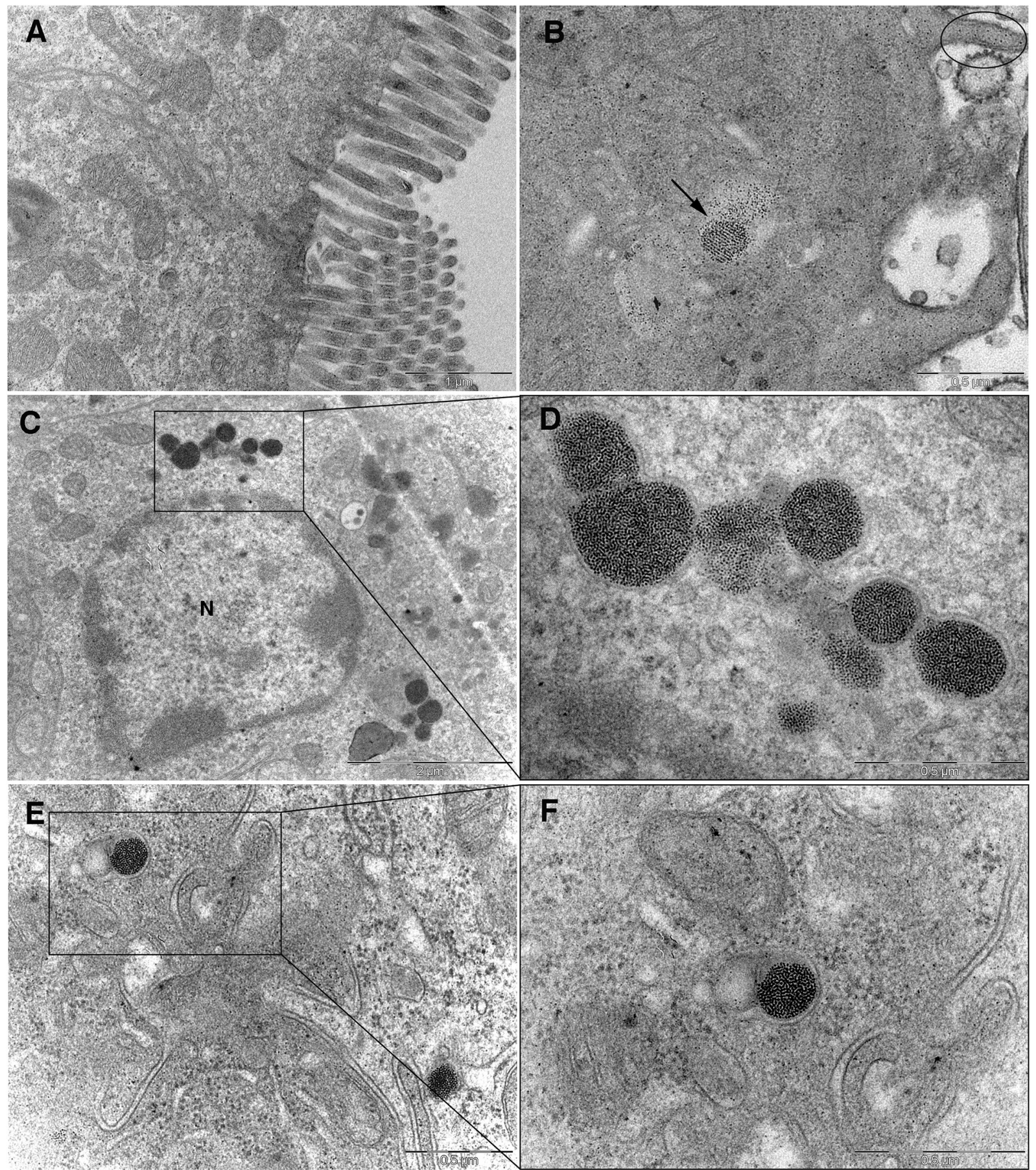

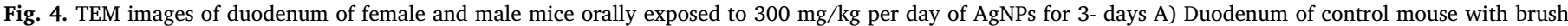

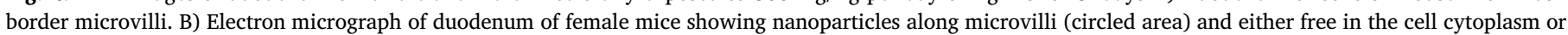

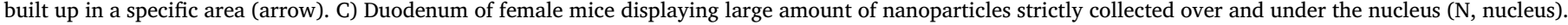

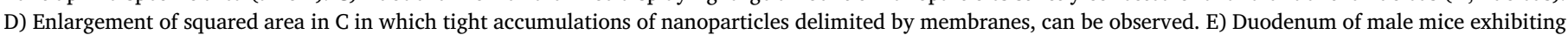
an aggregation of nanoparticles within a membrane bound structure as can be better appreciated in D (enlargement of squared area in C).

liver, kidney and spleen even after acute, short-term oral exposure both in male and female mice, indicating that the AgNPs were absorbed and distributed in target tissues without marked differences between sexes. TEM analysis of liver and duodenum of Ag300 male and female mice clearly identified AgNPs either freely dispersed in the cytoplasm or enclosed in membrane bound structures, but no AgNPs were observed in the nucleus. Indeed, as suggested by Patlolla A.K., the AgNPs of $20 \mathrm{~nm}$ diameter can block the access to nuclear pores and the entrance in nucleus (Patlolla et al., 2015a). Moreover, accumulation of AgNPs in specific organelles, as peroxisome or lysosomes, can reduce the possibility that free NPs enter the nucleus (McShan and Ray, 2014).

The highest AgNPs content was found in emptied duodenum and the result was coherent with a previous study in rats orally treated for 28 days with $10 \mathrm{ml} / \mathrm{kg}$ bw of AgNP, size $14 \pm 4 \mathrm{~nm}$ in diameter, stabilized in aqueous suspension of polyvinylpyrrolidone (Loeschner et al., 2011).

In 2016 Hendrickson et al. studied biodistribution of non-coated AgNPs (12 nm of diameter) after single and repeated administration 

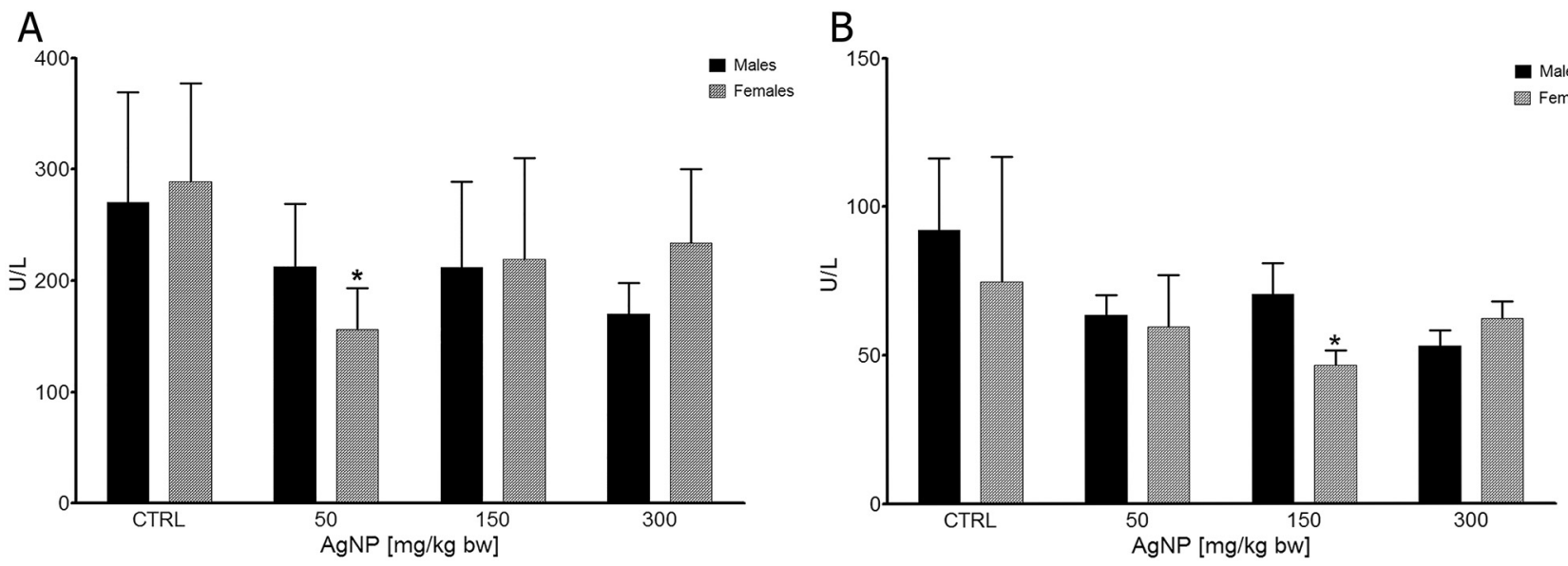

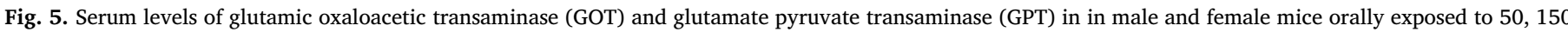
and $300 \mathrm{mg} / \mathrm{kg}$ per day of AgNPs for 3 days. ${ }^{*} \mathrm{P}<0.05$.

(30 days) at 2000 and $250 \mathrm{mg} / \mathrm{kg}$ bw, respectively; interestingly, after a single high-dose administration, the AgNPs were progressively moved (1 day, 7 and 14 days) from the deposition site organs (stomach and small intestine) towards other sites (liver, kidneys, spleen) (Hendrickson et al., 2016). Rats treated for 13 weeks with AgNPs (10, 75 , and $110 \mathrm{~nm}$ ) at 9,18 , and $36 \mathrm{mg} / \mathrm{kg}$ bw showed AgNPs predominantly located within cells of all the analyzed tissues (Boudreau et al., 2016). Differently from the present study, sex differences were noted in the pattern of distribution with higher concentration recorded in kidney, liver, jejunum and colon of female rats. In general, available studies on orally administered $\mathrm{Ag}$ in different forms (particulate or ionic) showed that it was deposited in all tissues and the concentration was dependent on dimensions and on exposure time. Oral administration of $90 \mathrm{mg} \mathrm{AgNPs} / \mathrm{kg}$ bw/day for 28 days (15 and $20 \mathrm{~nm}$ ) to rats localized the majority of the $\mathrm{Ag}$ in the stomach and small and large intestines, followed by (in descending order) liver, spleen, testes, kidneys, brain, lungs, blood, bladder and heart (van der Zande et al., 2012). Conversely, in rats orally dosed by gavage with $20 \mathrm{mg} / \mathrm{kg}$ bw AgNPs $(15.8 \pm 0.2 \mathrm{~nm})$ for $24 \mathrm{~h}$, NPs were exclusively detected in the feces, colon content and, to a minor extent, in the colon tissue (Juling et al., 2016). Indeed, in the present work, the biodistribution pattern can be explained with the short treatment duration, leading to higher concentrations in duodenum, the first contact site. Subsequently, AgNPs were absorbed and, through blood, where a high AgNP concentration was present, they were transported in other systemic organs as deposition site.
Despite the evident absorption and distribution, AgNPs did not affect general toxicological parameters at all the dose levels tested. Indeed, considering the widespread and long-term use of AgNPs as dietary supplement, no overt toxicity has been expected. Literature data already evidenced no signs of general toxicity and although animals were treated with lower doses, no effects on general parameters were recorded in short-, long- and chronic studies irrespective to AgNP diameters (Boudreau et al., 2016). No general toxicity was seen in Sprague-Dawley rats orally administered by gavage for 28 days with 0,30 , 300 and $1000 \mathrm{mg} / \mathrm{kg}$ bw/day of AgNPs (60 nm) (Kim et al., 2008) or in rats exposed for 90 days at $0,50,100$ and $200 \mathrm{mg} / \mathrm{kg}$ /day of PVPAgNPs (Garcia et al., 2016). Concerning biomarkers of hepatic and renal functions, isolated alterations in liver were present in female mice only, and in kidney in Ag300 male mice. Both endpoints were not supported by any histopathological and/or genotoxic alterations. For this reason, liver data were considered independent from the treatment. In kidney, since BUN was slightly but not significantly increased also in Ag150 group, this data might be considered as an early, treatment-related effect of renal damage.

Genomic stability after AgNP exposure was explored in a wide range of works by in vivo and in vitro models. In vivo genotoxic effects are often considered controversial because of uncertainties in key parameters such as size of nanoparticles, presence and type of coating, route and time of exposure.

Data generated by the present study can be considered reliable since the methodology was coherent with the recommended approach to
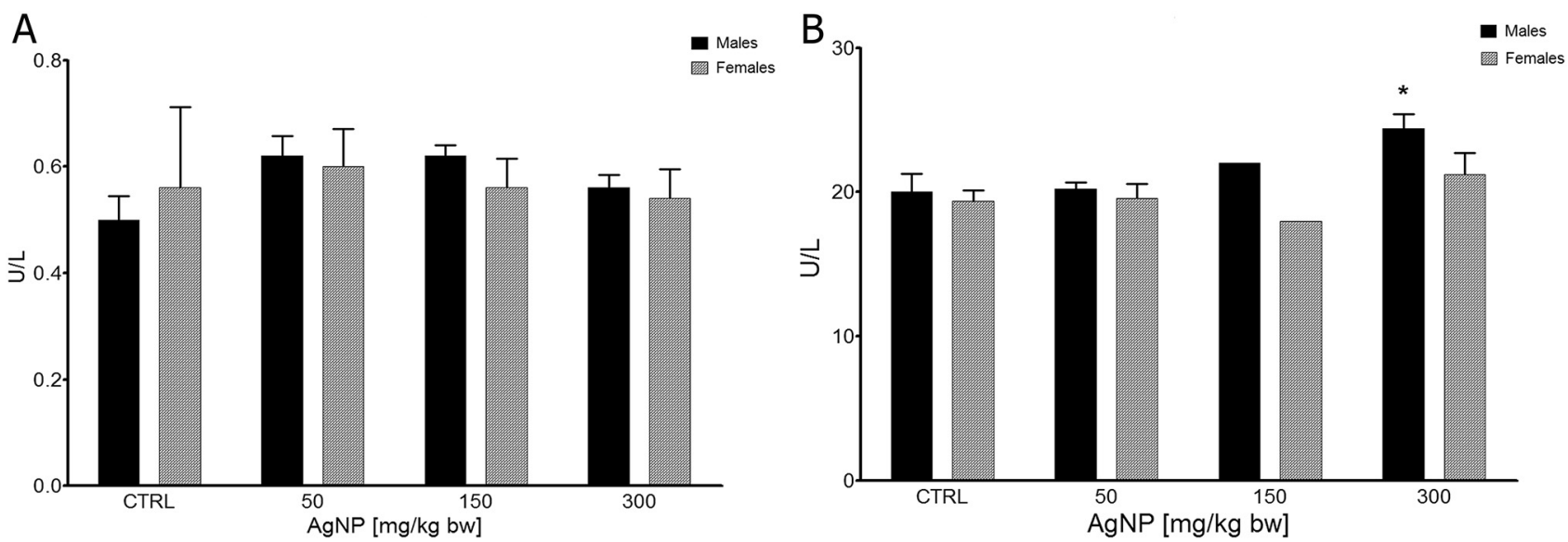

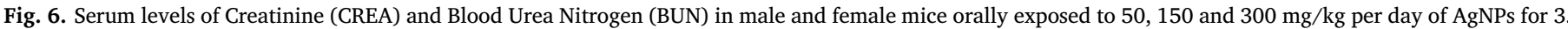
${ }^{*} \mathrm{P}<0.05$ 


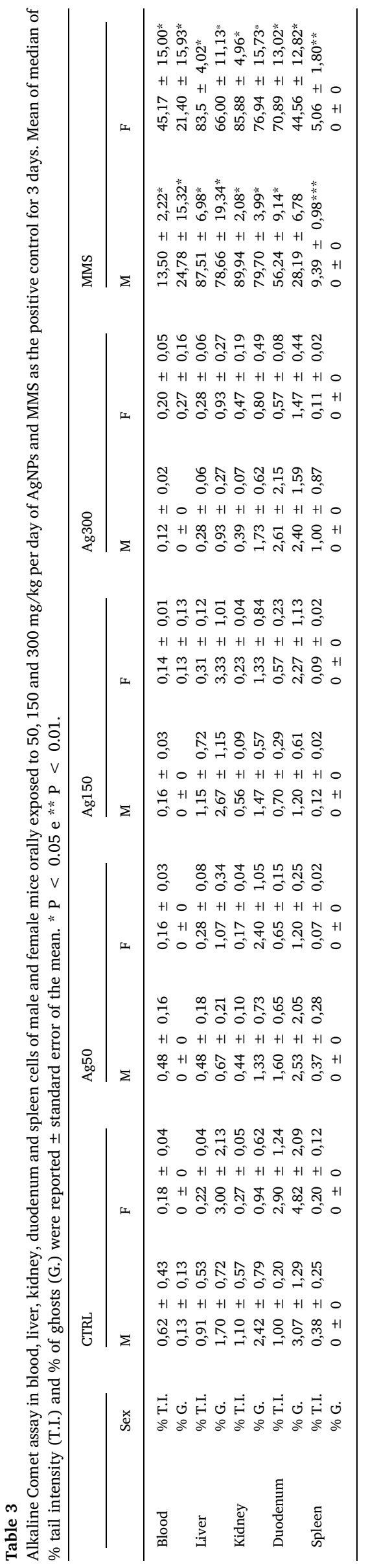

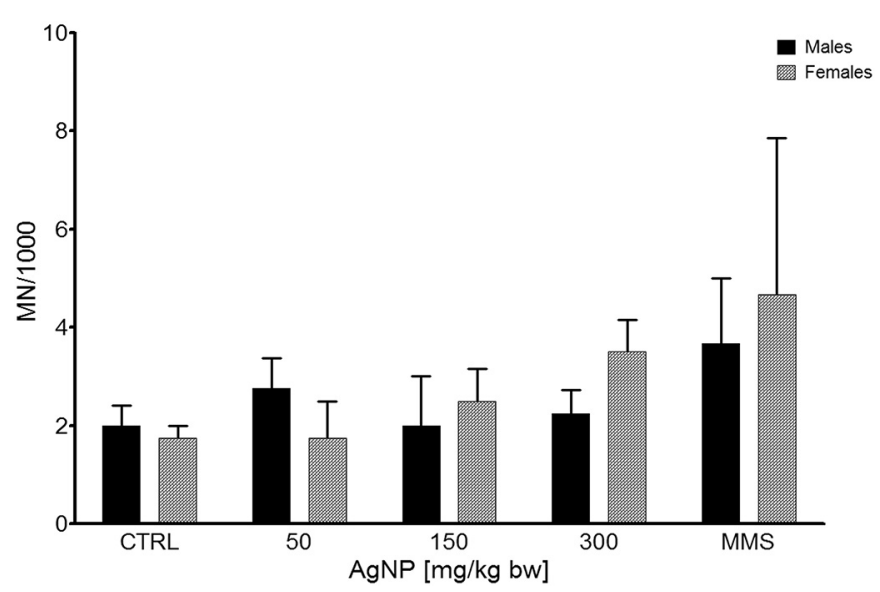

Fig. 7. Frequency of micronuclei in lymphocytes derived from murine spleen cells cultured for $44 \mathrm{~h}$ ex vivo of male and female mice orally exposed to 50, 150 and $300 \mathrm{mg} / \mathrm{kg}$ per day of AgNPs and MMS as the positive control for 3 days.

assess genotoxicity of NMs described by Elespuru et al. (2018), and the applied assays fall within the additional tests to be used when NMs are systemically available or when their presence in target tissues has been demonstrated.

In fact, although the absorption and distribution of AgNPs in target organs have been confirmed, no genotoxic damage - measured by Comet assay - has been recorded in all the selected tissues both in male and female mice. No noticeable adverse genotoxic effects have been also recorded in rats exposed via oral gavage to $0.5 \mathrm{mg} / \mathrm{kg} /$ day AgNPs for 45 days (Martins Jr et al., 2017), or treated daily by oral gavage for 13 weeks with $\operatorname{AgNPs}(10,75$, and $110 \mathrm{~nm})$ at 9,18 , and $36 \mathrm{mg} / \mathrm{kg}$ bw (Boudreau et al., 2016). Conversely, significantly increased genotoxic effects, i.e., number of structural chromosome aberrations, the frequency of micro-nucleated cells, damaged DNA as well as the induction of reactive oxygen species (ROS) were recorded in rats treated once a day for five days with doses of 5, 25, 50, 100, mg/Kg of AgNPs $10 \mathrm{~nm}$ diameter; the authors suggested that smaller particles are more likely than larger particles to release silver ions from the surface (Patlolla et al., 2015b). As for other nanosized particles of the same elemental composition (EFSA Scientific Committee et al., 2018), the physicochemical characteristics, including size, can lead to different biological activities, thus affecting the outcome of hazard identification.

TEM analysis showed the presence of AgNPs into the cells of liver and duodenum, in agreement with several previous works (Boudreau et al., 2016; Loeschner et al., 2011; Garcia et al., 2016). In fact, AgNPs were never located into the nucleus and this could explain the absence of genotoxic damages. Similar results were obtained by Martins Jr et al. (2017): male Wistar rats orally treated with AgNPs and TiO2 NPs, individually or in mixture, at $0.5 \mathrm{mg} / \mathrm{kg}$ bw day for 45 days, showed no DNA damage by Comet assay in blood and liver cells; AgNPs resulted in oxidative stress (Martins Jr et al., 2017). In 2014, Huk et al. suggested that AgNPs can induce DNA damage by indirect mechanism mediated by ROS production and indicated by DNA oxidation or by direct contact with DNA (Huk et al., 2014). Indeed, in aqueous solutions, AgNPs can dissolute in $\mathrm{Ag}$ ions $\left(\mathrm{Ag}^{+}\right)$which are mainly responsible of the toxicity induced by AgNPs (McShan and Ray, 2014) and massive amount of $\mathrm{Ag}^{+}$improves ROS production (Beer et al., 2012). In this work, although a direct measure of potential $\mathrm{Ag}^{+}$formation has not been performed, the AgNP dispersions had $\mathrm{pH} 7$ and in this condition the formation of $\mathrm{Ag}^{+}$ions was certified to be minimal (De Matteis et al., 2015). Since a direct contact of AgNPs with the nucleus has been excluded, and no genotoxicity has been recorded, this can be considered an indirect evidence of no $\mathrm{Ag}^{+}$ion formation in the present conditions. Moreover, validation test performed before the biodistribution analysis suggested that AgNPs or the $\mathrm{Ag}^{+}$ion recovery were similar in different 
tissues and the potential dissolution of AgNPs in $\mathrm{Ag}^{+}$ions may be excluded.

Micronucleus assay performed on spleen lymphocytes showed no differences among groups. AgNPs $(60 \mathrm{~nm})$ administered to male and female rats over a period of 28 days at 30,300 and $1000 \mathrm{mg} / \mathrm{kg} / \mathrm{bw}$ per day, according to OECD test guideline 407 protocol, evidenced no significant difference in the micronuclei polychromatic erythrocytes (MN PCEs) of bone marrow (Kim et al., 2008). In the present conditions, the absence of AgNPs in the nucleus can account for the lack of genotoxicity both as DNA breakage or MN formation.

\section{Conclusions}

After three-day oral acute exposure in adult mice of both sexes, biodistribution data indicate that AgNPs $(20 \mathrm{~nm})$ were mostly accumulated in duodenum - as first contact site - and subsequently transferred in liver, kidney and spleen by systemic circle. TEM analysis showed AgNPs free in the cytoplasm or accumulated in membrane bound structures but never in the nucleus, accounting for the lack of genotoxic damage evaluated by Comet or Micronucleus assays. Overall, the in vivo genotoxicity data generated on the basis of the OECD Guideline 489 provided new insight for a comprehensive risk assessment of AgNPs by the oral route.

\section{CRediT authorship contribution statement}

Laura Narciso:Conceptualization, Investigation, Data curation, Writing - original draft, Writing - review \& editing.Lucia Coppola:Investigation, Data curation, Writing - original draft, Writing - review \& editing.Gabriele Lori:Investigation, Data curation, Writing - original draft, Writing - review \& editing.Cristina Andreoli:Investigation, Data curation, Writing - original draft, Writing - review \& editing.Andrea Zjino:Investigation, Data curation, Writing - original draft, Writing - review \& editing.Beatrice Bocca:Investigation, Data curation, Writing - original draft, Writing review \& editing.Francesco Petrucci:Investigation, Data curation, Writing - original draft, Writing - review \& editing.Antonio Di Virgilio:Investigation.Andrea Martinelli:Investigation, Data curation.Antonella Tinari:Investigation, Data curation, Writing original draft, Writing - review \& editing.Francesca Maranghi:Conceptualization, Methodology, Writing - original draft, Writing - review \& editing, Supervision, Project administration, Funding acquisition.Roberta Tassinari:Conceptualization, Methodology, Investigation, Data curation, Writing - original draft, Writing - review \& editing, Visualization.

\section{Declaration of Competing Interest}

The authors declare that they have no known competing financial interests or personal relationships that could have appeared to influence the work reported in this paper.

\section{Acknowledgements}

This work has been supported by the project FILAS-RU-2014-1041 funded by Italian Lazio RegionL.R. 13/2008.

\section{Appendix A. Supplementary data}

Supplementary data to this article can be found online at https:// doi.org/10.1016/j.impact.2020.100221.

\section{References}

Asare, N., Duale, N., Slagsvold, H.H., Lindeman, B., Olsen, A.K., Gromadzka-Ostrowska, J., et al., 2016. Genotoxicity and gene expression modulation of silver and titanium dioxide nanoparticles in mice. Nanotoxicology 10, 312-321.

Azqueta, A., Gutzkow, K.B., Brunborg, G., Collins, A.R., 2011. Towards a more reliable comet assay: Optimising agarose concentration, unwinding time and electrophoresis conditions. Mutation Research/Genetic Toxicology and Environmental Mutagenesis 724, 41-45.

Beer, C., Foldbjerg, R., Hayashi, Y., Sutherland, D.S., Autrup, H., 2012. Toxicity of silver nanoparticles - nanoparticle or silver ion? Toxicol. Lett. 208, 286-292.

Behra, R., Sigg, L., Clift, M.J., Herzog, F., Minghetti, M., Johnston, B., et al., 2013. Bioavailability of silver nanoparticles and ions: from a chemical and biochemical perspective. J.R. Soc. Interface. 10, 20130396.

Blanco, J., Tomas-Hernandez, S., Garcia, T., Mulero, M., Gomez, M., Domingo, J.L., et al., 2018. Oral exposure to silver nanoparticles increases oxidative stress markers in the liver of male rats and deregulates the insulin signalling pathway and p53 and cleaved caspase 3 protein expression. Food Chem. Toxicol. 115, 398-404.

Bocca, B., Sabbioni, E., Micetic, I., Alimonti, A., Petrucci, F., 2017. Size and metal composition characterization of nano- and microparticles in tattoo inks by a combination of analytical techniques. J. Anal. At. Spectrom. 3.

Bocca, B., Caimi, S., Senofonte, O., Alimonti, A., Petrucci, F., 2018. ICP-MS based methods to characterize nanoparticles of $\mathrm{TiO} 2$ and $\mathrm{ZnO}$ in sunscreens with focus on regulatory and safety issues. Sci. Total Environ. 630, 922-930.

Boudreau, M.D., Imam, M.S., Paredes, A.M., Bryant, M.S., Cunningham, C.K., Felton, R.P., et al., 2016. Differential effects of silver nanoparticles and silver ions on tissue accumulation, distribution, and toxicity in the Sprague Dawley rat following daily oral gavage administration for 13 weeks. Toxicol. Sci. 150, 131-160.

Caldecott, K.W., 2008. Single-strand break repair and genetic disease. Nat. Rev. Genet. 9, 619-631.

Calderon-Jimenez, B., Johnson, M.E., Montoro Bustos, A.R., Murphy, K.E., Winchester, M.R., Vega Baudrit, J.R., 2017. Silver nanoparticles: technological advances, societal impacts, and metrological challenges. Front. Chem. 5, 6.

Carlessi, L., Fusar Poli, E., Bechi, G., Mantegazza, M., Pascucci, B., Narciso, L., et al., 2014. Functional and molecular defects of hiPSC-derived neurons from patients with ATM deficiency. Cell Death Dis. 5, e1342.

Choi, J.I., Chae, S.J., Kim, J.M., Choi, J.C., Park, S.J., Choi, H.J., et al., 2018. Potential silver nanoparticles migration from commercially available polymeric baby products into food simulants. Food Addit Contam. Part A. Chem. Anal. Control.Expo.Risk Assess. 35, 996-1005.

De Matteis, V., Malvindi, M.A., Galeone, A., Brunetti, V., De Luca, E., Kote, S., et al., 2015 Negligible particle-specific toxicity mechanism of silver nanoparticles: the role of $\mathrm{Ag}$ + ion release in the cytosol. Nanomedicine 11, 731-739.

EFSA Panel on Contaminants in the, Food Chain, 2015. Scientific opinion on nitrofurans and their metabolites in food. EFSA J. 13, 4140.

EFSA Panel on Food Additives and Nutrient Sources added, to Food, 2016. Scientific opinion on the re-evaluation of silver (E 174) as food additive. EFSA J. 14, 4364.

Elespuru, R., Pfuhler, S., Aardema, M.J., Chen, T., Doak, S.H., Doherty, A., et al., 2018. Genotoxicity assessment of nanomaterials: recommendations on best practices, assays, and methods. Toxicol. Sci. 164, 391-416.

ML Fernández-Cruz, D.C Hernández-Moreno, J. Cross, R. K. Stockmann-Juvala, 03, VR Lopes, M Matzke, et al., Quality evaluation of human and environmental toxicity studies performed with nanomaterials - the GUIDEnano approach. Environ. Sci. Nano, 2018,5, 381-397. 5 (2018) 381-397.

Garcia, T., Lafuente, D., Blanco, J., Sanchez, D.J., Sirvent, J.J., Domingo, J.L., et al., 2016. Oral subchronic exposure to silver nanoparticles in rats. Food Chem. Toxicol. 92, 177-187.

Glei, M., Schneider, T., Schlormann, W., 2016. Comet assay: an essential tool in toxicological research. Arch. Toxicol. 90, 2315-2336.

Hartemann, P., Hoet, P., Proykova, A., Fernandes, T., Baun, A., De Jong, W., et al., 2015 Nanosilver: safety, health and environmental effects and role in antimicrobial resistance. Mater. Today 18, 122-123.

Hayashi, M., 2016. The micronucleus test-most widely used in vivo genotoxicity test. Genes Environ. 38 (2016) 18-016-0044-x. eCollection.

Hendrickson, O.D., Klochkov, S.G., Novikova, O.V., Bravova, I.M., Shevtsova, E.F. Safenkova, I.V., et al., 2016. Toxicity of nanosilver in intragastric studies: biodistribution and metabolic effects. Toxicol. Lett. 241, 184-192.

Huk, A., Izak-Nau, E., Reidy, B., Boyles, M., Duschl, A., Lynch, I., et al., 2014. Is the toxic potential of nanosilver dependent on its size? Part Fibre Toxicol 11, 65-14-65-1.

Juling, S., Bachler, G., Gotz, N. von, Lichtenstein, D., Bohmert, L., Niedzwiecka, A., et al., 2016. In vivo distribution of nanosilver in the rat: the role of ions and de novo-formed secondary particles. Food Chem. Toxicol. 97, 327-335.

Karlsson, H.L., Di Bucchianico, S., Collins, A.R., Dusinska, M., 2015. Can the comet assay be used reliably to detect nanoparticle-induced genotoxicity? Environ. Mol. Mutagen. $56,82-96$.

Kim, D.W., Hong, G.H., Lee, H.H., Choi, S.H., Chun, B.G., Won, C.K., et al., 2007. Effect of colloidal silver against the cytotoxicity of hydrogen peroxide and naphthazarin on primary cultured cortical astrocytes. Int.J.Neurosci. 117, 387-400.

Kim, Y.S., Kim, J.S., Cho, H.S., Rha, D.S., Kim, J.M., Park, J.D., et al., 2008. Twenty-eightday oral toxicity, genotoxicity, and gender-related tissue distribution of silver nanoparticles in Sprague-Dawley rats. Inhal. Toxicol. 20, 575-583.

Liu, J., Hurt, R.H., 2010. Ion release kinetics and particle persistence in aqueous nanosilver colloids. Environ. Sci. Technol. 44, 2169-2175.

Liu, J., Wang, Z., Liu, F.D., Kane, A.B., Hurt, R.H., 2012. Chemical transformations of nanosilver in biological environments. ACS Nano 6, 9887-9899.

Loeschner, K., Hadrup, N., Qvortrup, K., Larsen, A., Gao, X., Vogel, U., et al., 2011. Distribution of silver in rats following 28 days of repeated oral exposure to silver nanoparticles or silver acetate. Part Fibre Toxicol 8, 18-8977-8-18.

Maranghi, F., Tassinari, R., Narciso, L., Tait, S., Rocca, C.L., Felice, G.D., et al., 2018. In Vivo Toxicity and Genotoxicity of Beauvericin and Enniatins. Combined Approach to 
Study In Vivo Toxicity and Genotoxicity of Mycotoxins Beauvericin (BEA) and Enniatin B (ENNB). 15. EFSA Supporting Publications, pp. 1406E.

Martins Jr., A.D.C., Azevedo, L.F., de Souza Rocha, C.C., Carneiro, M.F.H., Venancio, V.P., de Almeida, M.R., et al., 2017. Evaluation of distribution, redox parameters, and genotoxicity in Wistar rats co-exposed to silver and titanium dioxide nanoparticles. J. Toxicol. Environ. Health A. 80, 1156-1165.

McShan, D., Ray, P.C., Yu, H., 2014. Molecular toxicity mechanism of nanosilver. J. Food Drug Anal. 22, 116-127.

Narciso, L., Parlanti, E., Racaniello, M., Simonelli, V., Cardinale, A., Merlo, D., et al., 2016. The response to oxidative DNA damage in neurons: mechanisms and disease. Neural Plast 2016, 3619274.

OECD, 2016a. Test No. 489: In Vivo Mammalian Alkaline Comet Assay. https://doi.org/ 10.1787/9789264264885-en. ed., https://www.oecd-ilibrary.org/content/ publication/9789264264885-en.

OECD, 2016b. Test No. 474: Mammalian Erythrocyte Micronucleus Test.

Patlolla, A.K., Hackett, D., Tchounwou, P.B., 2015a. Genotoxicity study of silver nanoparticles in bone marrow cells of Sprague-Dawley rats. Food Chem. Toxicol. 85, $52-60$.

Patlolla, A.K., Hackett, D., Tchounwou, P.B., 2015b. Silver nanoparticle-induced oxidative stress-dependent toxicity in Sprague-Dawley rats. Mol. Cell. Biochem. 399, 257-268.

Romero, F., Rodriguez-Iturbe, B., Parra, G., Gonzalez, L., Herrera-Acosta, J., Tapia, E., 1999. Mycophenolate mofetil prevents the progressive renal failure induced by $5 / 6$ renal ablation in rats. Kidney Int. 55, 945-955.

SC, E.F.S.A., Hardy, A., Benford, D., Halldorsson, T., Jeger, M.J., Knutsen, H.K., et al., 2018. Guidance on risk assessment of the application of nanoscience and nanotechnologies in the food and feed chain: part 1, human and animal health. EFSA J. 16, e05327.

SCCS. Scientific Committee on Consumer Safety, 2018. OPINION ON Colloidal Silver (nano), EU publications. SCCS/1596/18 Final.

Singh, N.P., McCoy, M.T., Tice, R.R., Schneider, E.L., 1988. A simple technique for quantitation of low levels of DNA damage in individual cells. Exp. Cell Res. 175, $184-191$.

Tassinari, R., Cubadda, F., Moracci, G., Aureli, F., D’Amato, M., Valeri, M., et al., 2014 Oral, short-term exposure to titanium dioxide nanoparticles in Sprague-Dawley rat: focus on reproductive and endocrine systems and spleen. Nanotoxicology 8, 654-662. Tassinari, R., La Rocca, C., Stecca, L., Tait, S., De Berardis, B., Ammendolia, M.G., et al., 2015. In vivo and in vitro toxicological effects of titanium dioxide nanoparticles on small intestine. AIP Conference Proceedings 1667, 20016.

Tassinari, R., Narciso, L., Tait, S., Busani, L., Martinelli, A., Virgilio, A.D., et al., 2020. Juvenile toxicity rodent model to study toxicological effects of Bisphenol A (BPA) at dose levels derived from Italian children biomonitoring study. Toxicol. Sci. 173 (2), 387-401.

Ullah, A., Pirzada, M., Jahan, S., Ullah, H., Turi, N., Ullah, W., et al., 2018. Impact of lowdose chronic exposure to bisphenol A and its analogue bisphenol B, bisphenol $\mathrm{F}$ and bisphenol S on hypothalamo-pituitary-testicular activities in adult rats: a focus on the possible hormonal mode of action. Food Chem. Toxicol. 121, 24-36.

Verleysen, E., Van Doren, E., Waegeneers, N., De Temmerman, P.J., Abi Daoud Francisco, M., Mast, J., 2015. TEM and SP-ICP-MS analysis of the release of silver nanoparticles from decoration of pastry. J. Agric. Food Chem. 63, 3570-3578.

Yabuki, A., Suzuki, S., Matsumoto, M., Nishinakagawa, H., 1999. Morphometrical analysis of sex and strain differences in the mouse nephron. J. Vet. Med. Sci. 61, 891-896.

van der Zande, M., Vandebriel, R.J., Van Doren, E., Kramer, E., Herrera Rivera, Z., Serrano-Rojero, C.S., et al., 2012. Distribution, elimination, and toxicity of silver nanoparticles and silver ions in rats after 28-day oral exposure. ACS Nano 6, 7427-7442. 